\title{
Use of Focused Pulse-Echo Ultrasonics for Nondestructive Inspection of Thick Carbon-Carbon Structures
}

\author{
Dennis ROACH, Phil WALKINGTON, Sandia National Labs, New Mexico, U.S.A.
}

\begin{abstract}
The reinforced carbon-carbon (RCC) heat shield components on the Space Shuttle's wings must withstand harsh atmospheric reentry environments where the wing leading edge can reach temperatures of $3,000^{\circ} \mathrm{F}$. Potential damage includes impact damage, micro cracks, oxidation in the silicon carbide-to-carbon-carbon layers, and interlaminar disbonds. The thick, carbon-carbon and silicon-carbide layers in the heat shield panels are difficult to inspect and existing techniques were deemed to be inadequate. Since accumulated damage in these structures can lead to catastrophic failure of the Shuttle's heat protection system, it became imperative for NASA to quickly develop an acceptable health monitoring program. NASA selected a multi-method approach for inspecting the wing leading edge which includes eddy current, thermography, and ultrasonics. Sandia Labs produced the in-situ ultrasonic pitch-catch inspection method. Optimum combinations of custom ultrasonic probes and data anlaysis were merged into the overall inspection method needed to properly survey the heat shield panels. Comprehensive validation tests revealed that the ultrasonic pitch-catch inspection system is capable of reliably finding flaws of 0.25 ” diameter or greater in the heat shields. The inspection system is now in use at NASA to complete "Return-to-Flight" certification inspections prior to each Shuttle launch.
\end{abstract}

\section{Introduction}

After the loss of the Columbia Orbiter vehicle during a Shuttle Transport System mission (STS-117) in 2003, the Columbia Accident Investigation Board was formed to investigate the accident and make recommendations to increase system safety prior to return to flight (RTF). One of the chief recommendations was: Develop and implement a comprehensive inspection plan to determine the structural integrity of all Reinforced Carbon-Carbon system components. This inspection plan should take advantage of advanced nondestructive inspection (NDI) technology [1].

Rather than relying on computer-based analysis and extrapolated experience to reduce risk, the inspection plan called for recertification of the Shuttle for upcoming missions by performing an in-depth assessment of the Space Shuttle hardware. Previously, in situ NDI of the Reinforced Carbon-Carbon (RCC) heat shields consisted of visual and light touch tactile inspections. The accident investigation, along with complimentary NASA studies, determined that multiple forms of damage mechanisms could be missed by these inspections. The array of RCC damage scenarios include impact damage, loss of coating integrity on the outer surfaces, carbon cracks/fracture, interply delaminations, disbonds at the silicon-carbide to carbon-carbon interface, and silicon-carbide craze cracks. Many of these flaws are internal to the structure and are not visually evident.

Figure1 shows the RCC heat shields along the leading edge of the wings and on the nose of the Orbiter. Figure 2 includes several cross-section views of the RCC. The outer silicon- 
carbide (SiC) layer provides the thermal protection during Shuttle re-entry to the atmosphere while the carbon-carbon (C-C) layer provides the structural integrity for the panels. Figure 2 shows how micro-cracks can form in the Si-C layer and lead to one of the primary damage mechanisms: silicon-carbide craze cracking and subsequent subsurface oxidation which produces mass loss and reduction in the $\mathrm{C}-\mathrm{C}$ strength.

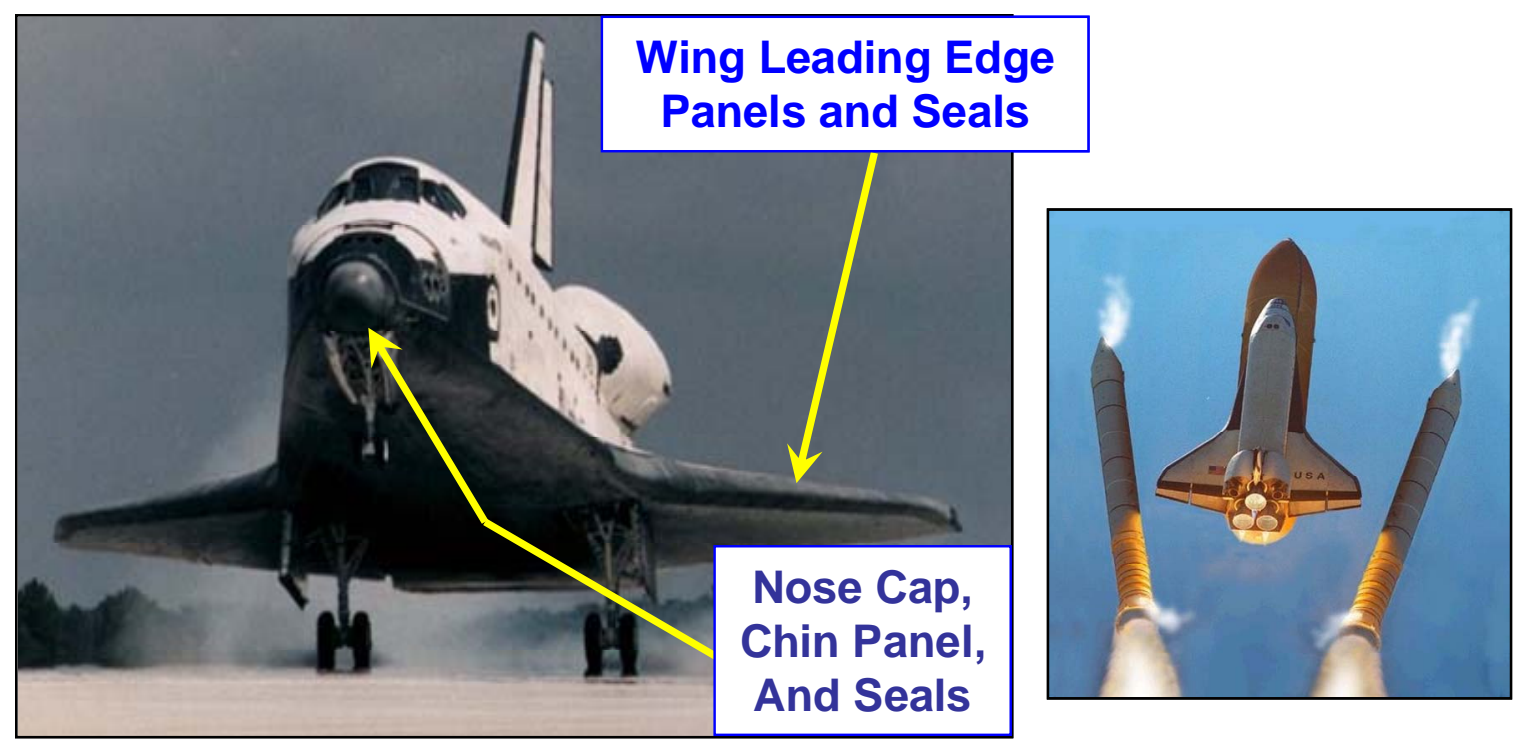

Figure 1: Reinforced Carbon-Carbon Heat Shields Along the Leading Edge of the Wings and on the Nose of the Orbiter

The reinforced carbon-carbon (RCC) heat shield components on the Orbiter's wings must withstand harsh atmospheric reentry environments where the wing leading edge can reach temperatures of $3,000^{\circ} \mathrm{F}$. The Space Shuttle Accident Investigation Board concluded that damage in the Shuttle's heat shields allowed temperatures to rise drastically in the Shuttle wings. This caused the inner structure to melt and the Shuttle to break up during re-entry. The thick, carbon-carbon and silicon-carbide layers in the heat shield panels are difficult to inspect and existing techniques were deemed to be inadequate. The overall health monitoring objective was to develop a comprehensive NDE program for the in-situ health monitoring of Orbiter RCC components. The focused goal was to deploy a variety of NDI methods to detect any RCC degradation that may compromise performance. To pursue these goals, NASA conducted an aggressive, nationwide search for advanced nondestructive inspection (NDI) technology that could improve Shuttle health monitoring.

Since accumulated damage in the RCC can lead to catastrophic failure of the Shuttle's heat protection system, it became imperative for NASA to quickly develop an acceptable health monitoring program. NASA's goal was to obtain turnkey inspection systems that could certify the integrity of the Shuttle wing heat shields prior to each mission. Because of the possibility of damaging the heat shields during removal, the NDI devices had to be deployed without removing the leading edge panels from the wing. NASA selected a multi-method approach for inspecting the heat shields which includes eddy current, thermography, and ultrasonics. The complementary superposition of these three inspection techniques produces a rigorous Orbiter certification process that can reliably detect the array of flaws expected in the Shuttle's RCC heat shields. 


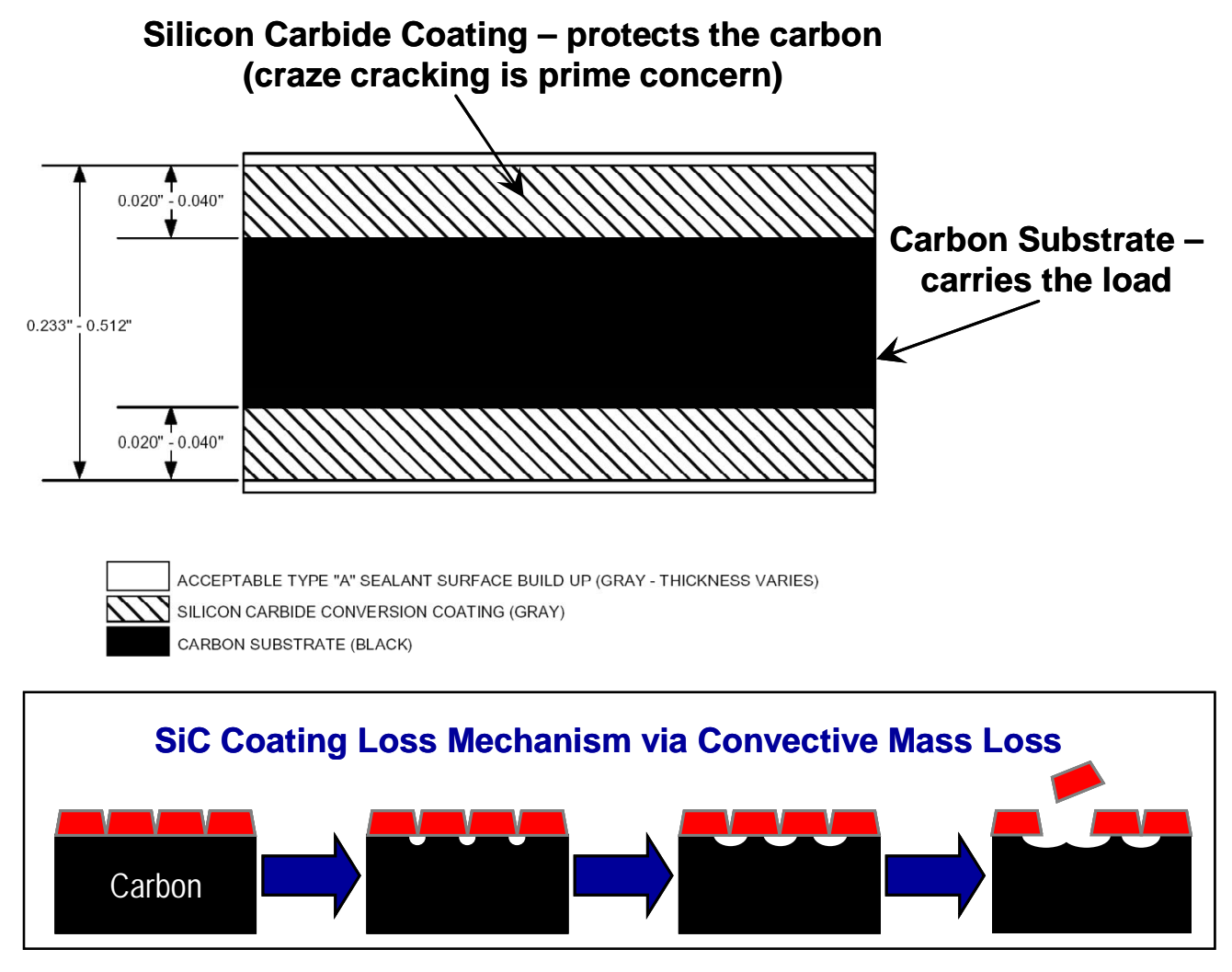

Figure 2: Composition of the RCC Panels and a Primary Damage Mechanism

An integrated team of participants from NASA-Johnson Space Center, NASA-Langley, NASA-Kennedy Space Center, Sandia National Laboratories, United Space Alliance (USA), Oceaneering Space Systems (OSS) and Boeing worked to develop and demonstrate nondestructive inspection (NDI) systems capable of assessing the RCC leading edge panels on-wing in the Orbiter Processing Facility (OPF). This team developed, tested, and evaluated an ultrasonic (UT), eddy current (EC), and thermography system to detect all potential flaws in the Shuttle RCC heat shields. Sandia Labs produced the in-situ ultrasonic inspection method while NASA Langley developed the eddy current and thermographic techniques. An extensive validation process, including blind inspections monitored by NASA officials, demonstrated the ability of these inspection systems to meet the accuracy, sensitivity, and reliability requirements. This paper presents the ultrasonic NDI development process [2]. The program included the use of flight hardware and scrap heat shield panels to discover and overcome the obstacles associated with damage detection in the RCC material. Optimum combinations of custom ultrasonic probes and data analysis were merged with the inspection procedures needed to properly survey the heat shield panels. System features were introduced to minimize the potential for human factors errors in identifying and locating the flaws. The ultrasonic inspection system successfully completed the certification process and is approved for health monitoring of the Shuttle Orbiter.

\section{Ultrasonic Inspection Method}

The principal reasons for exploring UT for the RCC inspections were the potential for: 1) superior penetrating power for detection of deep flaws, 2) high sensitivity permitting the detection of extremely small flaws, 3) accuracy in determining size and position of flaws, 4) completion of inspections using only the exterior accessible surface, 5) nonhazardous 
operations with no effect on personnel and equipment nearby, 6) a high degree of portability, and 7) ability to digitally process the data for archiving.

Inspection Requirements - Structural analysis and strength testing of RCC panels determined that flaws on the order of $1 / 4$ " in dia. need to be reliably detected with the inspection systems. These flaws may be anywhere within the thickness of the panels (thickness range of 0.25 " to 0.375”). Ease of deployment considerations required that the device be light weight, easy and fast to set up, and capable of navigating all contours on the RCC heat shields. This includes the tight 3" radius regions in the wing leading edge panels. Figure 3 shows a side view of an RCC leading edge panel along with the hardware that attaches the panel to the main spar of the Orbiter's wing. The inspections are implemented in the OPF so the inspection methods must be unaffected by other maintenance activities that may be occurring simultaneously in adjacent regions.

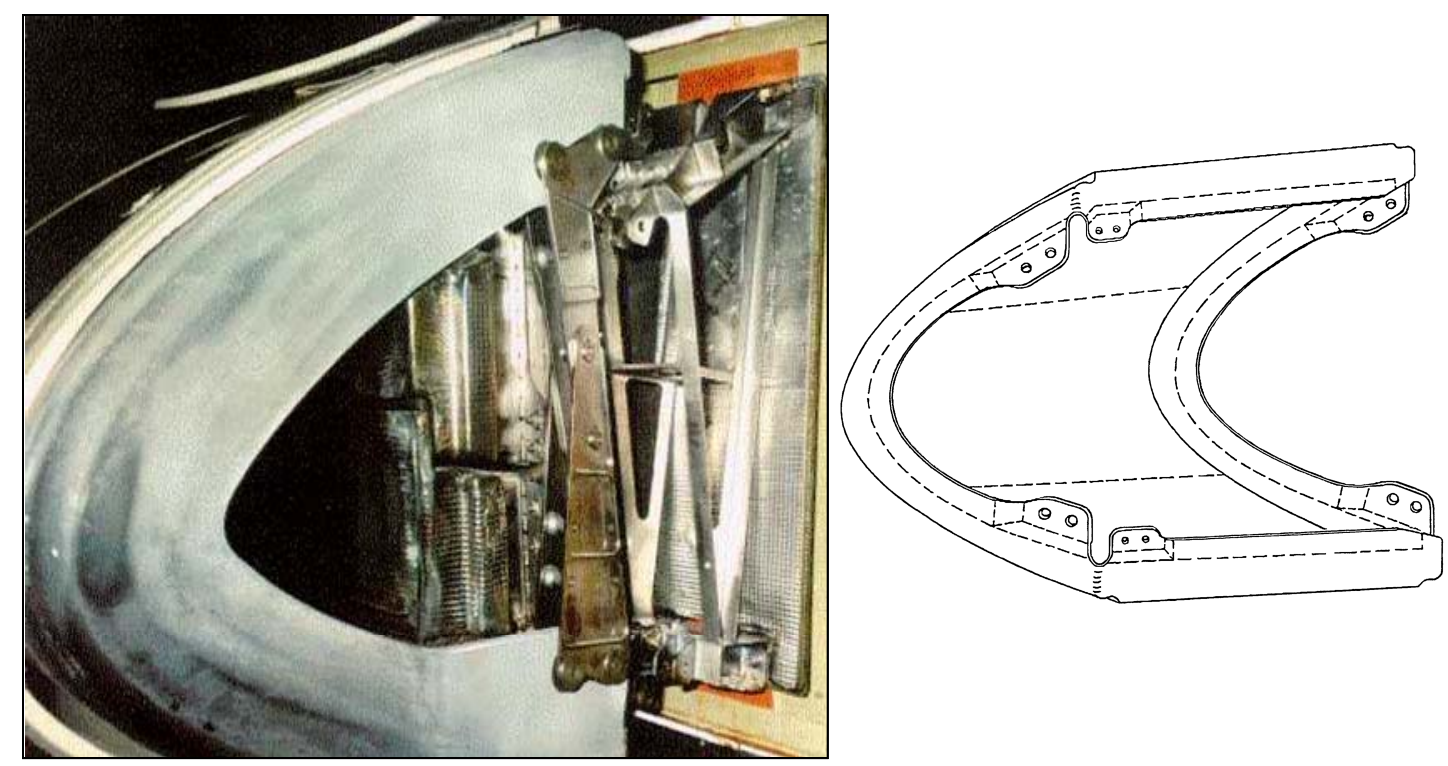

Figure 3: Geometry of the Wing Leading Edge Heat Shields and Mounting Hardware for Connection to Forward Spar of the Orbiter

Physics of Ultrasonics in RCC Material - The UT method introduces beams of high frequency sound waves into the RCC material for the detection of surface and subsurface flaws. The sound waves, at carefully optimized frequencies, travel through the material with some attendant loss of energy (attenuation) and are reflected at interfaces. The reflected beam is displayed and then analyzed to define the presence and location of flaws. The degree of reflection depends largely on the physical state of the materials forming the interface. Cracks, delaminations, shrinkage cavities, pores, disbonds, and other discontinuities that produce reflective interfaces can be detected. Complete reflection, partial reflection, scattering, or other detectable effects on the ultrasonic waves are used as the basis for flaw detection. In addition to wave reflection, other variations in the wave that are monitored include: time of transit through the test piece, attenuation, and features of the spectral response. The types of RCC flaws detectable by the ultrasonic method include cracks, delaminations, voids, local mass loss, global mass loss, and impact damage.

In UT $90^{\circ}$ pulse-echo inspections, a single transducer acts alternately as the sending and receiving transducer as shown in the Figure 4 schematic. This allows the interrogating apparatus to have the smallest possible footprint and optimize inspection coverage along the edges of the specimen or in areas of rapidly changing geometry. The mechanical vibration 
(ultrasound) is introduced into the test piece through a couplant and travels by wave motion through the specimen at the velocity of sound, which depends on the material. If the pulses encounter a reflecting surface, some or all of the energy is reflected and monitored by the transducer. The reflected beam, or echo, can be created by any normal or abnormal (flaw) interface. Figure 4 shows the interaction of UT waves with various interfaces within the RCC structure.

A-Scan signals, shown at the top of Figure 4, represent the response of the sound waves, in amplitude and time, as they travel through the material. As the waves interact with defects or flaw interfaces within the solid and portions of the pulse's energy are reflected back to the transducer, the flaws are detected, amplified and displayed. The interaction of the ultrasonic waves with defects and the resulting time vs. amplitude signal produced depends on the wave mode, its frequency and the material properties of the structure. Flaw size can be estimated by comparing the amplitude of a discontinuity signal with that of a signal from a discontinuity of known size and shape. Flaw location (depth) is determined from the position of the flaw echo along a calibrated time base.

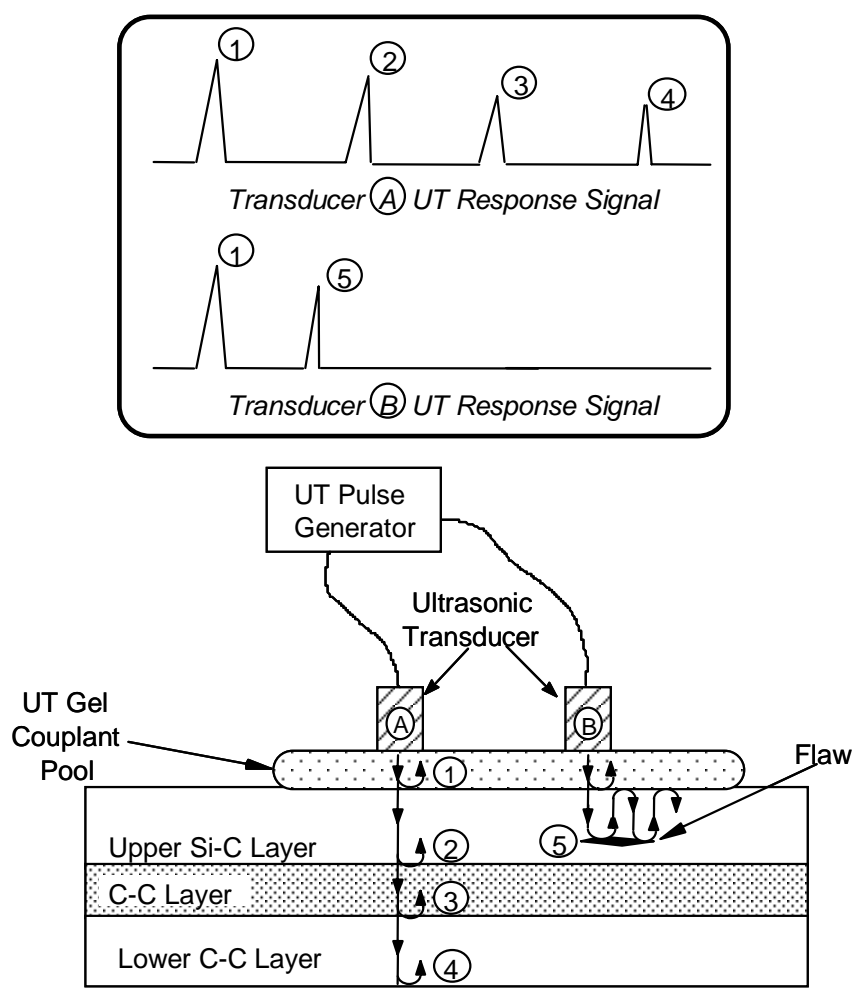

Figure 4: Schematic of Pulse-Echo Ultrasonic Inspection and Reflection of UT Waves at Assorted Interfaces

Wide Area Inspections Aided by C-Scan Technology - It is sometimes difficult to clearly identify flaws using ultrasonic A-Scan signals alone. Small porosity pockets commonly found in composites, coupled with signal fluctuations caused by material nonuniformities can create signal interpretation difficulties. Significant improvements in flaw detection can be achieved by taking the A-Scan signals and transforming them into a single C-Scan image of the part being inspected. C-scans are two-dimensional images (area maps) produced by digitizing the point-by-point signal variations of an interrogating sensor while it is scanned over a surface. A computer converts the point-by-point data into a color representation and 
displays it at the appropriate point in an image. Specific "gates" can be set within the data acquisition software to focus on response signals from particular regions within the structure. C-Scan area views provide the inspector with easier-to-use and more reliable data with which to recognize flaw patterns. This format provides a quantitative display of signal amplitudes or time-of-flight data obtained over an area. The X-Y position of flaws can be mapped and time-of-flight data can be converted and displayed by image processing equipment to provide an indication of flaw depth.

The robotic scanner system used by Sandia to inspect the RCC is shown in Figure 5. The scanning unit containing the UT transducer is moved over the surface of the test piece using a search pattern of closely spaced parallel lines. A mechanical linkage connects the scanning unit to $\mathrm{X}$-axis and $\mathrm{Y}$-axis position indicators which feed position data to the computer. The unit is tethered to a remotely located computer for control and data acquisition. The echo signal is recorded, versus its $\mathrm{X}-\mathrm{Y}$ position on the test piece, and a color coded image is produced from the relative characteristics of the sum total of signals received.

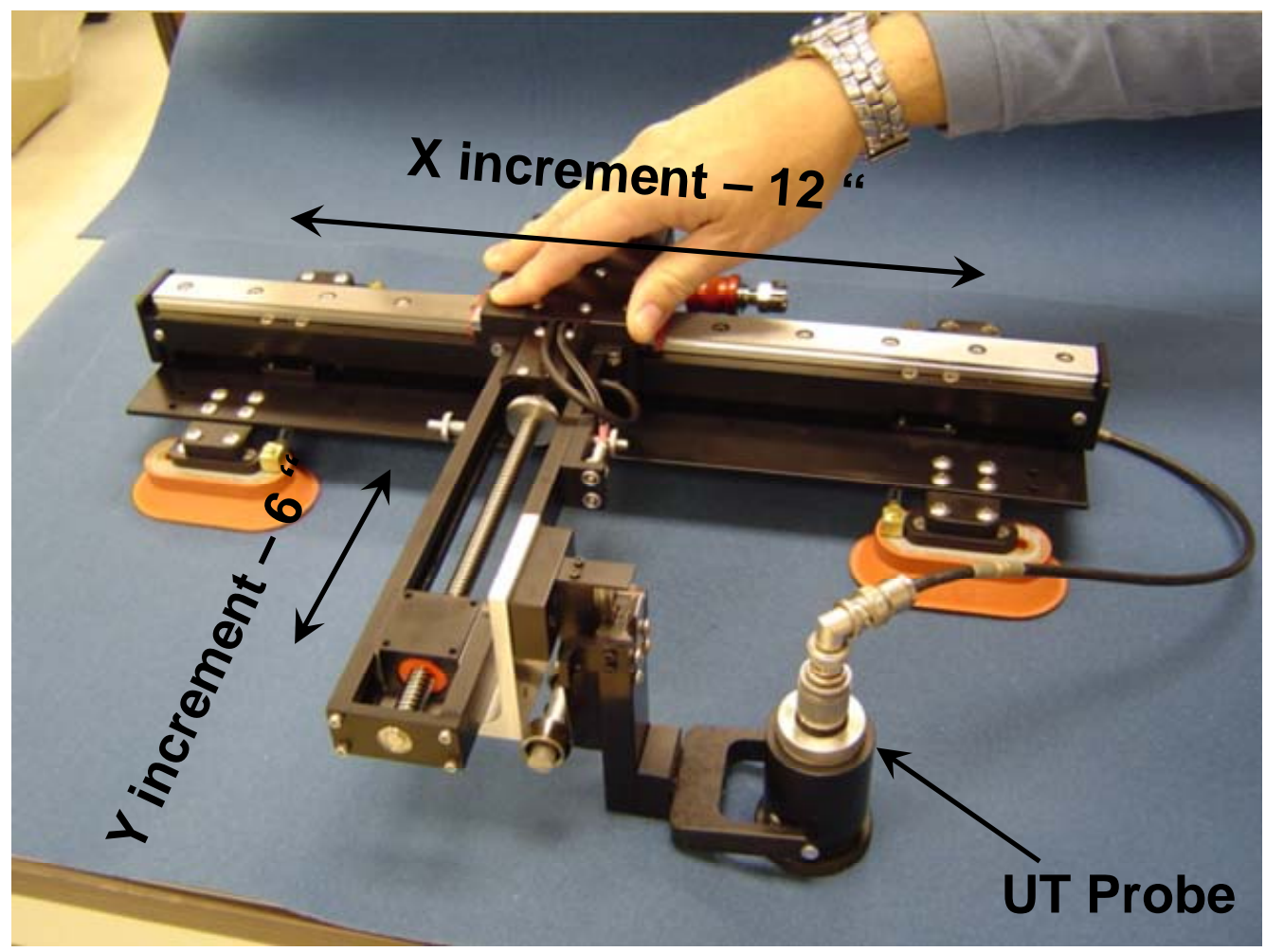

Figure 5: Pulse-Echo Ultrasonic NDI System with Robotic Scanner Device

Optimization of Ultrasonic Signal - In order to better accommodate the specific needs of the RCC panel inspections, custom devices were added to the inspection system. First, a gimbal device was developed to: 1) adapt to surface irregularities and varying slopes, and 2) maintain the transducer perpendicularity with the inspection surface. This minimizes loss of coupling and misdirection/loss of UT energy between the transducer and the inspection surface. Figure 6 shows a close-up view of the transducer assembly in the gimbal positioning mechanism (articulating yoke) which provided rotational degrees of freedom about the $\mathrm{X}$ and $\mathrm{Y}$ axis. Next, a linear spring was designed to provide proper transducer contact pressure and surface following in the contoured regions. The linear springs placed in the housing shown in Figure 6 provided a constant surface contact force of 3 lbs. (1.3 psi) over the entire 2” range of Z-axis (out-of-plane) motion. 
Another inspection system enhancement involved the use of a "weeper" system to optimize ultrasonic coupling into and out of the RCC material. Normal UT coupling is achieved through the use of a gel or other liquid medium which can: 1) conform to the time varying gap between the UT transducer and the test surface, and 2) transmit acoustic energy with minimum attenuation. In most UT inspections, this couplant is initially applied to the surface and then periodically replenished as the couplant is pushed away by the scanning motion. When proper coupling is lost, a corresponding loss of UT signal (signal "drop out") is observed. Once a scanner has been set in motion, a preset area is inspected and any signal drop-out areas must be revisited. In order to avoid this potentially time consuming process, an optimized and continuous source of UT coupling is needed.

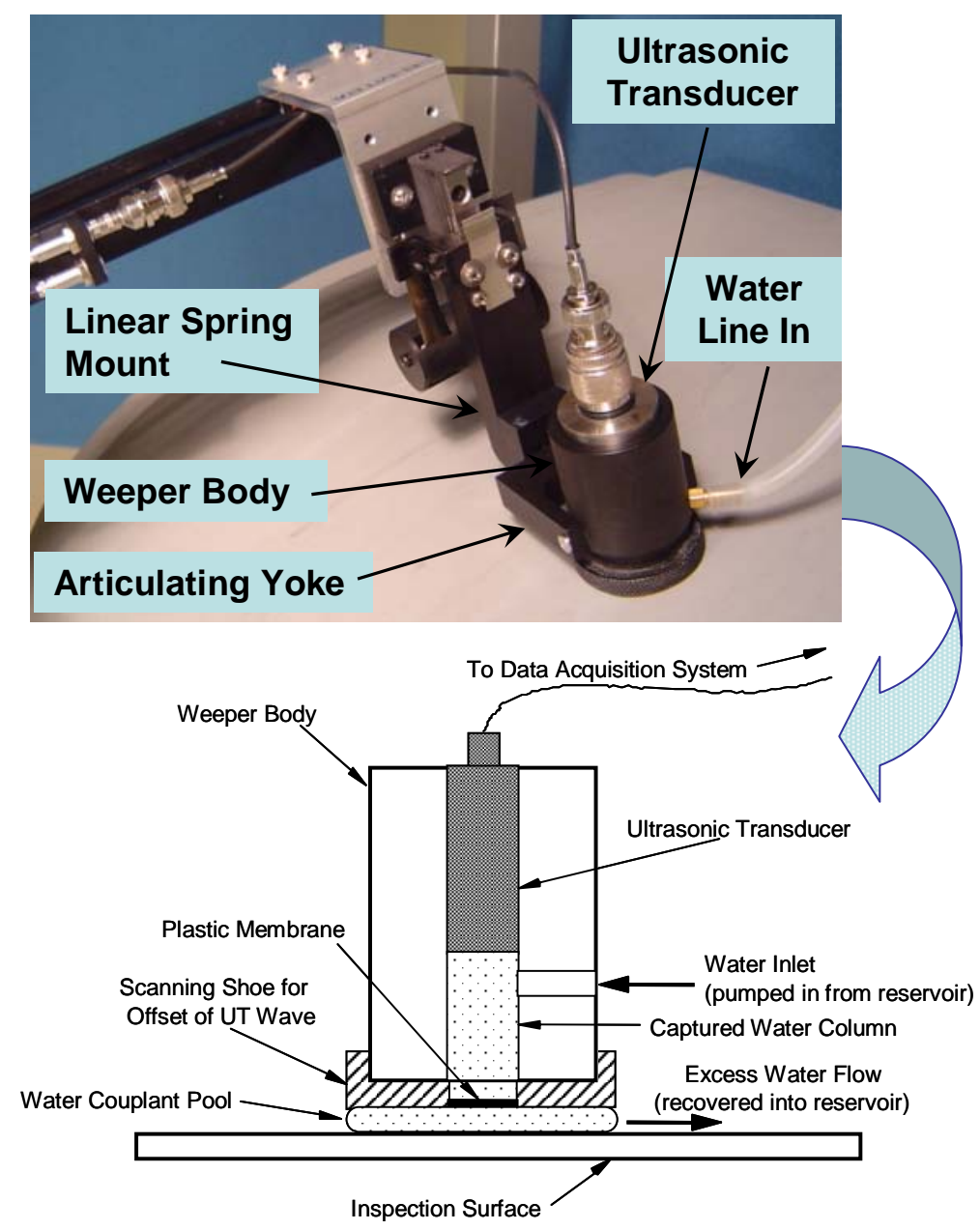

Figure 6: Schematic of Assembled Ultrasonic Sensor System - Weeper Body and Ultrasonic Transducer with Linear Spring for Surface Following

To achieve this, a weeper system was integrated into the UT system. The Figure 6 photograph and schematic show the array of parts that make up the complete assembly. The weeper system forms the transducer arm of the scanning unit and consists of a UT emitter/receiver transducer in a water column. The weeper body is a cylinder with a plastic membrane at the base. Within the weeper body, a column of water is contained between the membrane and the transducer as shown in Figure 6. The membrane is pierced several times to produce a suitable water flow between the membrane and the inspection surface. Water is continuously pumped into the weeper body in order to maintain the water column and the 
water couplant "pool" between the weeper body and the inspection surface. Water running away from the couplant "pool" can be recovered and returned to a reservoir to create a closed-loop pumping system. This water column and associated water pool beneath the membrane provides very uniform and consistent coupling for the ultrasonic waves moving into and out of the test article. This eliminates data irregularities, provides more energy at the inspection point, and produces better output signals (less dispersion). The end results are: 1) improved signal-to-noise (amplitude), 2) better time-of-flight data, and 3) greater flaw detection resolution. Experimentation revealed that the optimum signal was produced when the probe offset (water column length) was set to 1.1” - 1.2”.

Use of Customized, Focused Transducers with Pulse Echo Ultrasonics - The detection of disbonds at the various bond-line interfaces was quite challenging. Since the RCC material is highly attenuative, optimizing the UT probe design to produce the highest signal strength was critical. When using 1/2" diameter focus transducers, the echo amplitude change observed at the disbond was only slightly different than that caused by the unflawed portions of the composite bond-line. In order to find the best signal strength and clarity, experiments were conducted with a number of different transducer designs. Four different focal lengths were examined (1”, 2”, 3”, and 5”) and three different transducer diameters were explored (0.5”, 1 ", and 1.5”). Finally, a range of frequencies were used to conduct the inspections. In order to produce the depth of penetration desired, it was first determined that the frequency should be on the order of $1 \mathrm{MHz}$. The inspection frequency was adjusted in trial tests over the range of $1-2.5 \mathrm{MHz}$. A noticeable improvement in the bond-line echo response was obtained by using a 1" diameter, 2" focus transducer. In addition, it was determined that the $1 \mathrm{MHz}$ frequency provided the strongest and most uniform signal within the RCC material. Sandia worked with ultrasonic transducer manufacturers to produce the following custom probe for this inspection: $1 \mathrm{MHz}, 1$ " diameter, 2" focused beam, with a housing geometry that sealed tightly within the weeper body shown in Figure 6.

Use of UT Gates for Proper Data Acquisition - One of the key ingredients in completing a successful RCC inspection is the correct positioning of a series of gates corresponding to specific depths and amplitudes within the RCC. Data acquisition gates are an essential element in C-scan systems. They allow users to focus on specific phenomena. The gates operate on the A-scan signals received during an inspection and allow users to focus on specific phenomenon in particular time frames (depths) within the structure. User-specified gates allow only those echo signals that are received within a limited range of delay times and amplitudes following the initial pulse to be admitted to the receiver-amplifier circuit. The color coded C-scan reflects these focus areas. Depth and amplitude gates are adjustable. By setting gates for specific ranges, echo signals from key areas of the test article, parallel to the scanned surface, can be recorded. In these inspections, the gates were set so that front surface reflections from the RCC are de-emphasized in the display. Echoes from within the composite laminate, especially those at the front and back $\mathrm{Si}-\mathrm{C}$ to $\mathrm{C}-\mathrm{C}$ interface, were emphasized.

A typical A-scan trace from a composite laminate inspection is shown in Figure 7. It is labeled with the key UT wave interface areas and includes the three sample gates used in an inspection. Gate 1 is set to detect delaminations, porosity, or other flaws within the composite laminate. Normally, the amplitude of the UT waveform should be relatively small compared with the adjacent peaks (labeled "C" and "D") shown in Figure 7. If, however, a flaw is present, a large amplitude signal, which exceeds the gate \#1 level, will occur in this gate and create a delamination flaw map. Gates \#2 and \#3 are used to check the integrity of the bond/interface layers in the RCC panels. The loss of peak "D" at gate \#2 or peak "E" at gate 
\#3 would indicate a disbond or other loss of materials at the front or back Si-C to C-C interfaces, respectively. References [3-4] provide additional information on how the C-Scan technique and custom gate settings can aid in the interpretation of composite inspections.

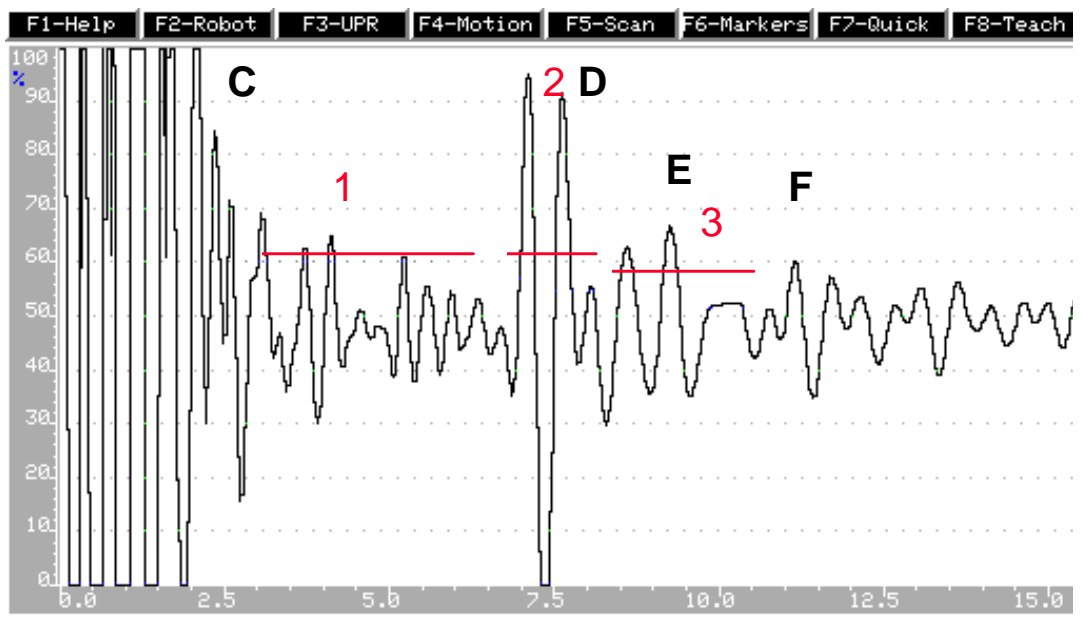

Figure 7: A-Scan Trace Showing Actual Response from a Thick Composite Laminate and the Location of Gates to Detect the Following:

Gate \#1 - Delamination or Porosity Signals, Gate \#2 - Bond Interface Signals, and Gate \#3 - Backwall Echo

\section{Peak C = Echo from the Front Surface of the RCC Panel \\ Peak D = Echo from Front Side Si-C to C-C Interface \\ Peak E = Echo from Front Side Si-C to C-C Interface Aluminum \\ Peak F = Echo from the Back Wall of the RCC Panel}

Data Analysis for Pulse-Echo Ultrasonic NDI Technique - Once the digitized A-scan waveforms are recorded during the ultrasonic pulse-echo inspection of the RCC material, the amplitude and time of flight peak signals can be displayed as a C-scan image and analyzed to determine if a flaw exists within the material. The reflected beam from the back surface of the RCC material can be used as the starting point for this analysis. The C-scan image can reveal several variations within the RCC material. The peak amplitude from the back surface is affected by the attenuation within the material and will be displayed in the pseudo colored C-scan image. Any large amplitude change $(>12 \mathrm{db})$ in the C-scan image is reported as a possible flaw. Depending upon the geometry of a flaw and location within the RCC material, the amplitude might not appear very different than that of the surrounding back surface. This is where the time of flight C-scan image can show a slight shift in the color of the back surface. By analyzing both pseudo colored images (amplitude and time of flight) and the Ascan waveforms, the inspector can determine if a flaw exists within the RCC material. The time of flight C-scan image also shows thickness variations such as those created by the taper along the edges of the RCC panel. When viewing the C-scan images it is possible to dynamically adjust the color palettes (color style and number of different color bins) corresponding to either the amplitude engaging the various gates or the time of flight for the signal to arrive at the gates. Rather than looking at the C-scan images in a static mode, it was determined that the dynamic display of data - produced by adjusting the color palettes in real time - provided the best approach for identifying flaws. By using this dynamic data analysis method, it was possible to study anomalies within the scans to determine if they were actually flaws or just other acceptable signal variations within the material. This approach also minimized the number of false calls. 
Deployment of UT Inspection System - In order to deploy the UT inspection equipment such that it could cover all required inspection regions of the heat shields, it was necessary to mate the scanner shown in Figure 5 with an adjustable positioning apparatus. The arrangement of work platforms and safe operation in the OPF produced the following deployment requirements: vertical reach of 46 " to 70 ", horizontal reach of 1 " to 18 ", inspections performed in upright (upper surface) and inverted (lower surface) modes, position locks to ensure inspection location, and hardware tie-downs to avoid damage to nearby Shuttle hardware. To address all of these requirements, the mechanism shown in Figure 8 was designed. It consists of a customized tripod and extension slide that was modified to mount the X-Y scanner. The slide mounts were designed to accommodate both upright and inverted scanning, as well as vertical scanning. All tripod degrees of freedom and the slide translation are equipped with locking devices to ensure a constant origin (0,0 start position) during the scanning process. This positioning mechanism can be adjusted to reach all RCC inspection areas. It is lightweight and can be rapidly set-up by a single worker. The tripod was stabilized through tie-downs that were engineered to connect the apparatus to any location on the OPF work platforms.
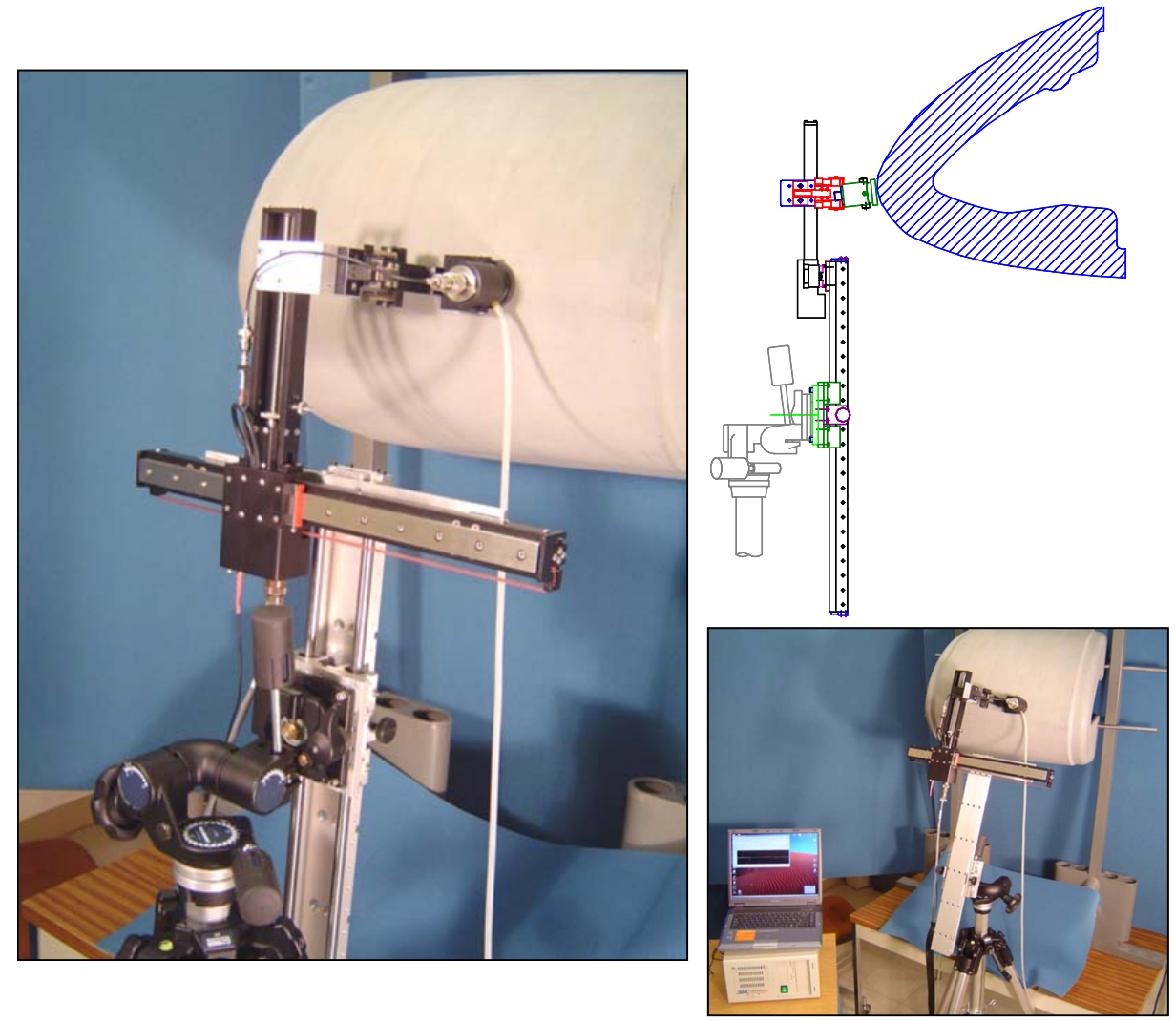

Figure 8: Ultrasonic System Components and Deployment on RCC Panel

To allow the UT method to produce its best possible results, it was also necessary to fabricate representative reference standards. The NDI methods are implemented by on-site personnel at KSC. This means that the UT system needs to be "turn-key" and able to demonstrate repeatable data acquisition. It must have some type of calibration that assures its proper operation as well as detection sensitivity to known "standards". RCC panels removed from existing Shuttle Orbiters were used to create such standards. They contained all inspection impediments (e.g. attenuation levels, geometry, access limitations) and realistic flaws. These NDI standards are used to calibrate the equipment prior to each use and to produce representative signals with which to characterize flaws. 


\section{Quantitative Performance Evaluation}

Using the ultrasonic scanner system and enhancements described above, a series of NDI validation tests were performed. The test specimens, representing the variations in RCC thickness and geometry, contained the full array of flaws that may exist in the heat shields. Flaws were engineered using specialized machining processes, localized loading, exposure to extreme temperatures, and impact damage produced by projectiles traveling at different speeds. The total validation process involved 46 test specimens that contained 306 flaws. Final system certification was achieved through blind inspections on a complete RCC wing leading edge panel and mating T-seal. Statistically valid, probability of flaw detection values were calculated, along with associated false call and flaw sizing information, to quantify the overall performance of the ultrasonic inspection method.

Figure 9 shows a sample RCC disk with damage produced by simulated exposure to re-entry conditions. First, a thin crack was machined into the Si-C layer. Then, a set of disks were placed in an arc jet and exposed to $3,000^{\circ} \mathrm{F}$ temperatures for various lengths of time. This produced different amounts of oxidation and decay of the $\mathrm{C}-\mathrm{C}$ material beneath the Si-C layer as shown in Figure 9. The schematic and photo in Figure 9 also show how the surface of the disk remained unchanged, however, hidden subsurface flaws were present at the Si-C to C-C interface. A typical image produced by the ultrasonic scan is shown in Figure 10 along with some A-scan signals comparing the good and flawed regions. Similar clarity was obtained for both nearside and far side flaw inspections (see Figure 9). This indicates that the UT method is able to penetrate to the backside interface and detect flaws through the entire thickness of the RCC.
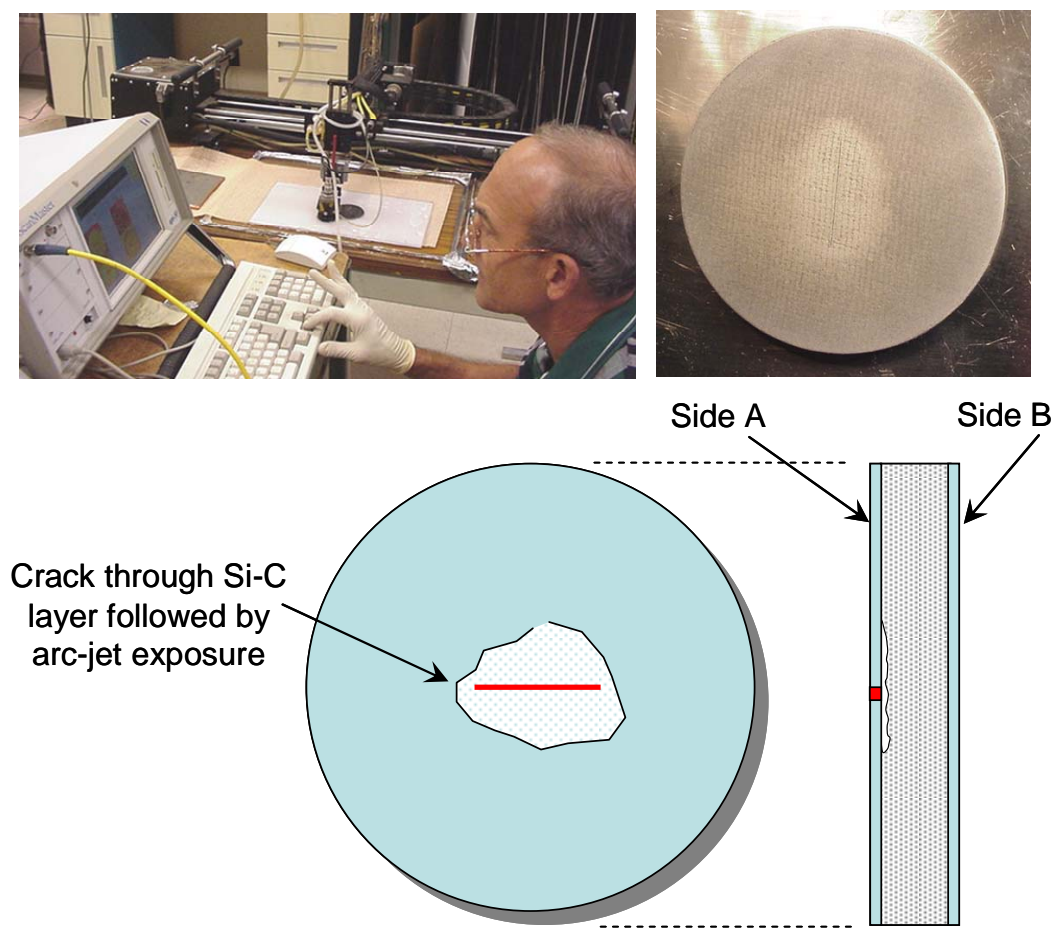

Figure 9: Arc Jet Disks with Induced Oxidation Damage in C-C Layer

- Inspection from Side A Simulates Nearside (Surface) Flaw Detection

- Inspection from Side B Simulates Backside (Deep) Flaw Detection 


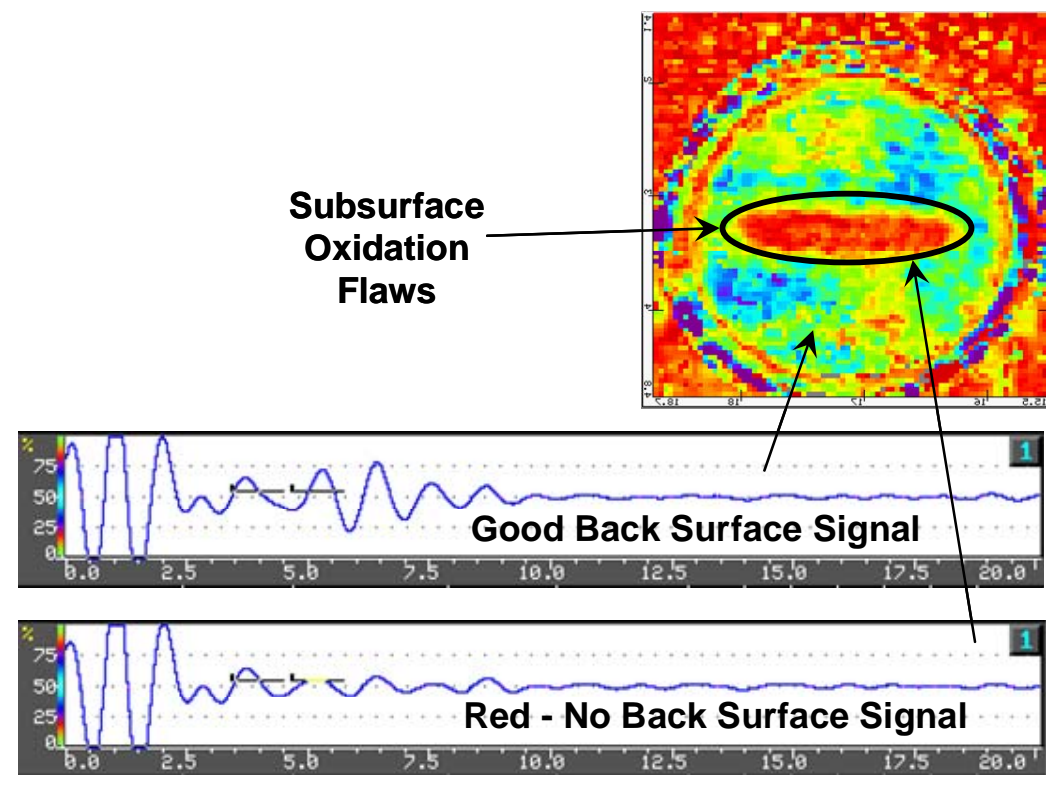

Figure 10: Sample C-Scan Image and A-Scan Signals Produced by UT Inspection System Revealing Subsurface Damage in Arc Jet Disks.

Figures 11 and 12 provide two examples of tests results from specimens containing machined flaws. The RCC panel in Figure 11 included flat bottom holes of different depths and diameters. This specimen was designed to assess flaw detection at each of the three critical depths and to determine the smallest possible flaw that can be found. The ultrasonic C-scan image on the right shows that all twelve flaws were detected. This demonstrates the ability of the UT system to detect flaws anywhere within the RCC thickness and reveals sensitivity down to a $1 / 8$ " diameter.

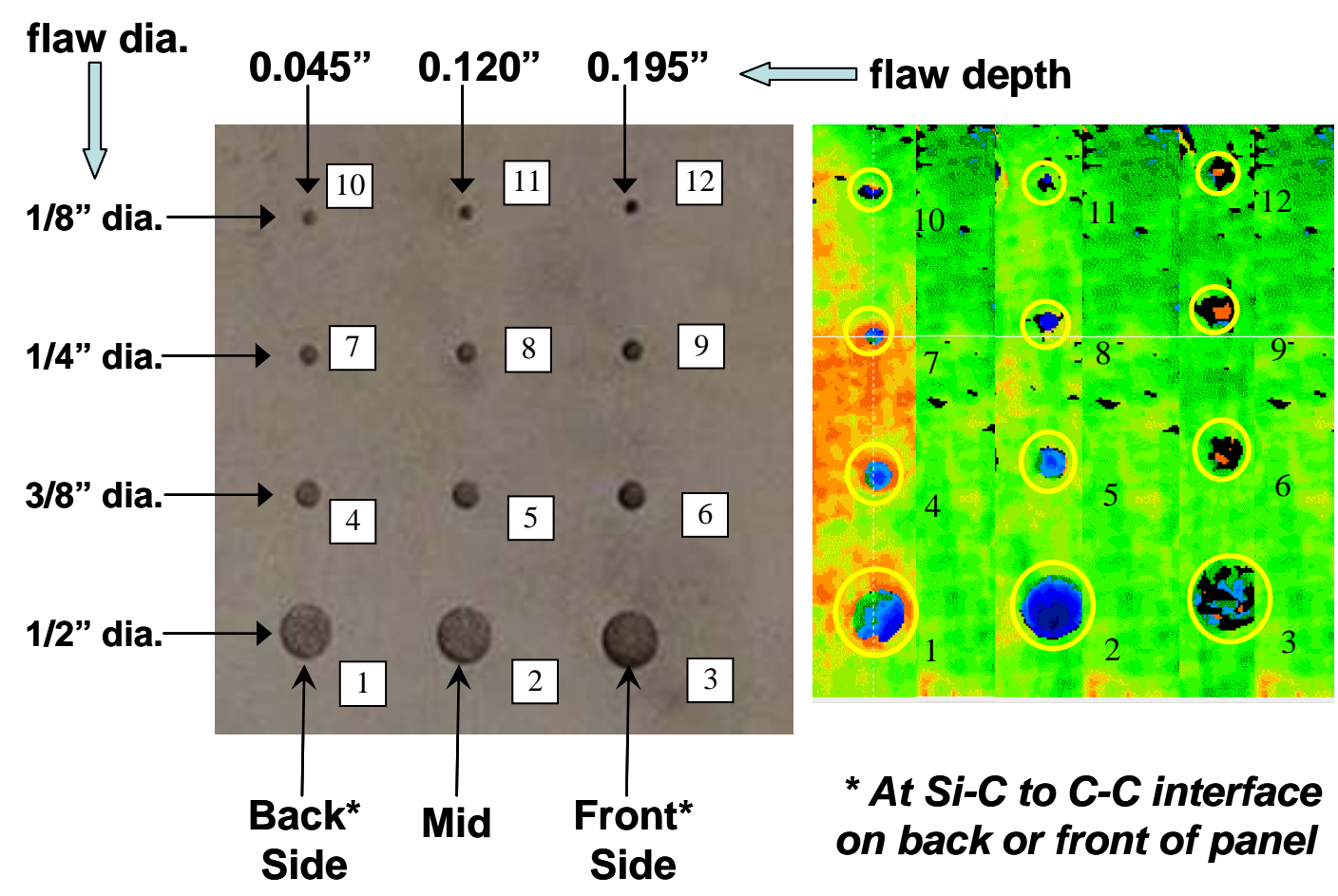

Figure 11: Photo of RCC Flight Hardware and Corresponding C-Scan Image of Flaws as Small as 1/8” Diameter at Different Depths in the Panel 
Another set of flawed specimens were produced using an electrical discharge machining (EDM) process. The sloped slots shown in Figure 12 were created by an EDM wire that was guided across and down through the RCC panel. The slots were used to determine the ability of NDI to detect flaws that are not contained at one level. Foreign object impact, for example, may induce a single damage site that includes multiple depths within the RCC material. The corresponding C-scan in Figure 12 illustrates that such flaws, as small as 1/8" in width, can be clearly imaged by the Sandia ultrasonic system. Another important example involves an RCC panel that was damaged in a natural way via impact. The photos in Figure 13 show that the front and back surfaces of the RCC panel do not have any visual indications of damage. However, internal damage was produced in the impact test. The C-scan in the lower portion of Figure 13 show how the internal damage is imaged by the UT inspection system.

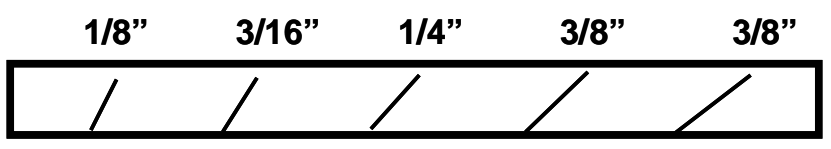

Side View
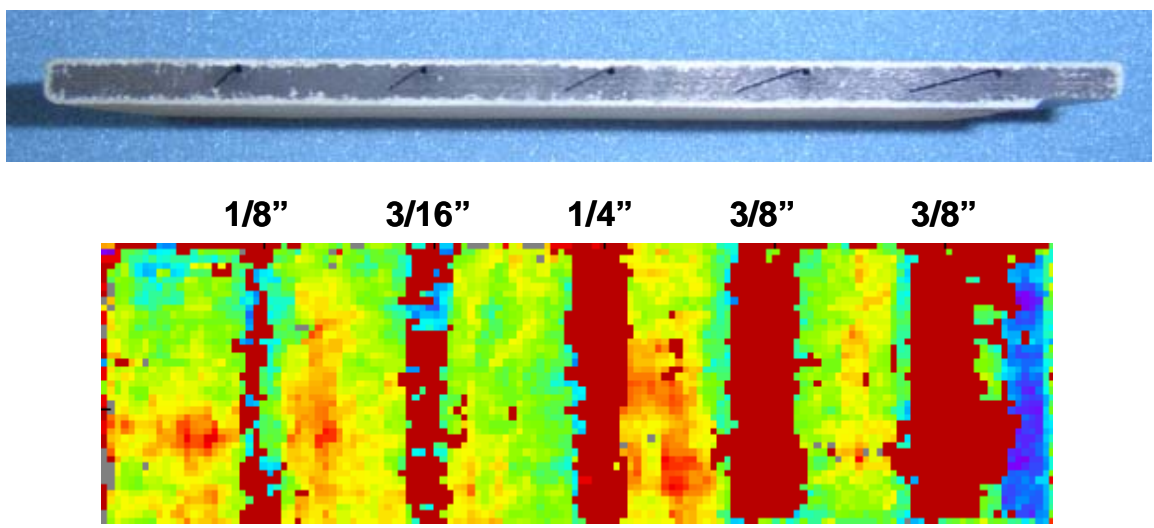

Top View C-scan

Figure 12: RCC Specimen with Sloped Slots and Time-of-Flight C-Scan Produced by the Ultrasonic Inspection System

90 degree impact at $2077 \mathrm{ft} / \mathrm{sec}$ - no visible damage on front or back side

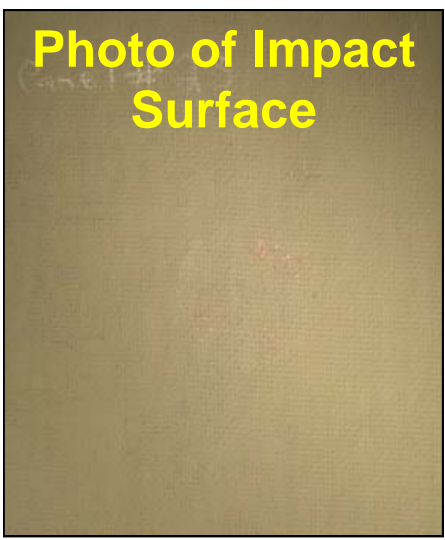

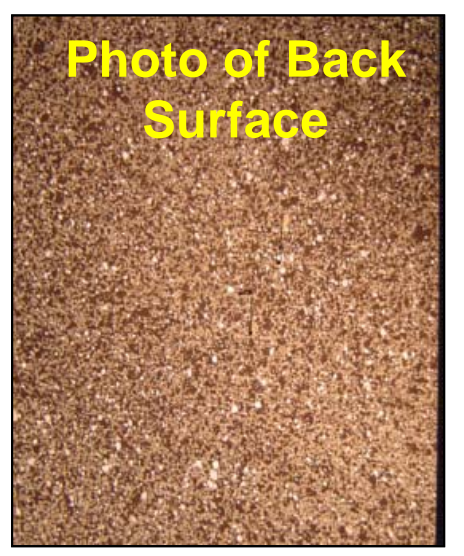

Pulse-Echo UT C-scan

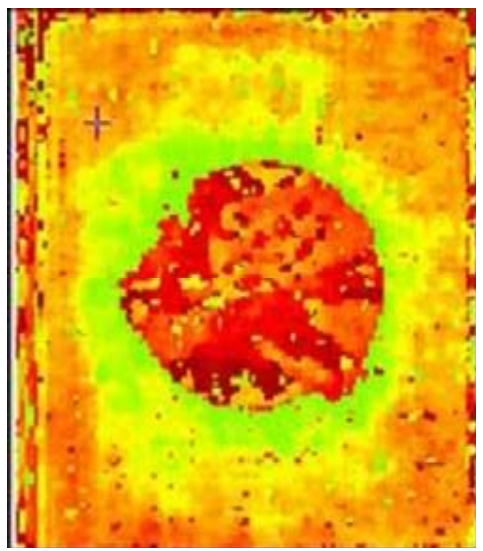

Figure 13: UT C-Scan Showing Subsurface Damage in NASA Impact Panels 
UT System Certification at NASA - Final validation and NASA certification of the UT inspection system was achieved through a series of carefully controlled inspections on several complete RCC panels including the one shown in Figure 14. The panels were mounted onto a test fixture to simulate heat shield accessibility, orientation, and other inspection impediments that will be encountered on the Space Shuttle Orbiter. The inspections were conducted in a blind fashion such that the inspectors had no knowledge of the flaws hidden within the RCC panel. All inspections were monitored by NASA officials in order to demonstrate the ability of the UT inspection system to meet the accuracy, sensitivity, and reliability requirements. The blind testing included 77 hidden flaws.

In general, the objectives of the validation and certification effort were to: 1) validate the functionality of the pulse-echo ultrasonic system and integrated scanner hardware by performing blind in situ inspection of a full scale RCC Orbiter Wing Leading Edge (WLE) Panel and mating Tee Seal, 2) quantitatively validate the ability of this inspection method to detect representative flaws ranging from 0.040 to 0.190 inches in depth and from 0.125 to 0.5 inches in diameter, 3) validate the ability of each system to accurately inspect hardware in a configuration that encompasses curvature and thickness variations representative of a RCC Panel (upper, lower, apex and thickness transition areas), and 4) validate that the inspection process is capable of meeting the applicable requirements of relevant NASA specifications.
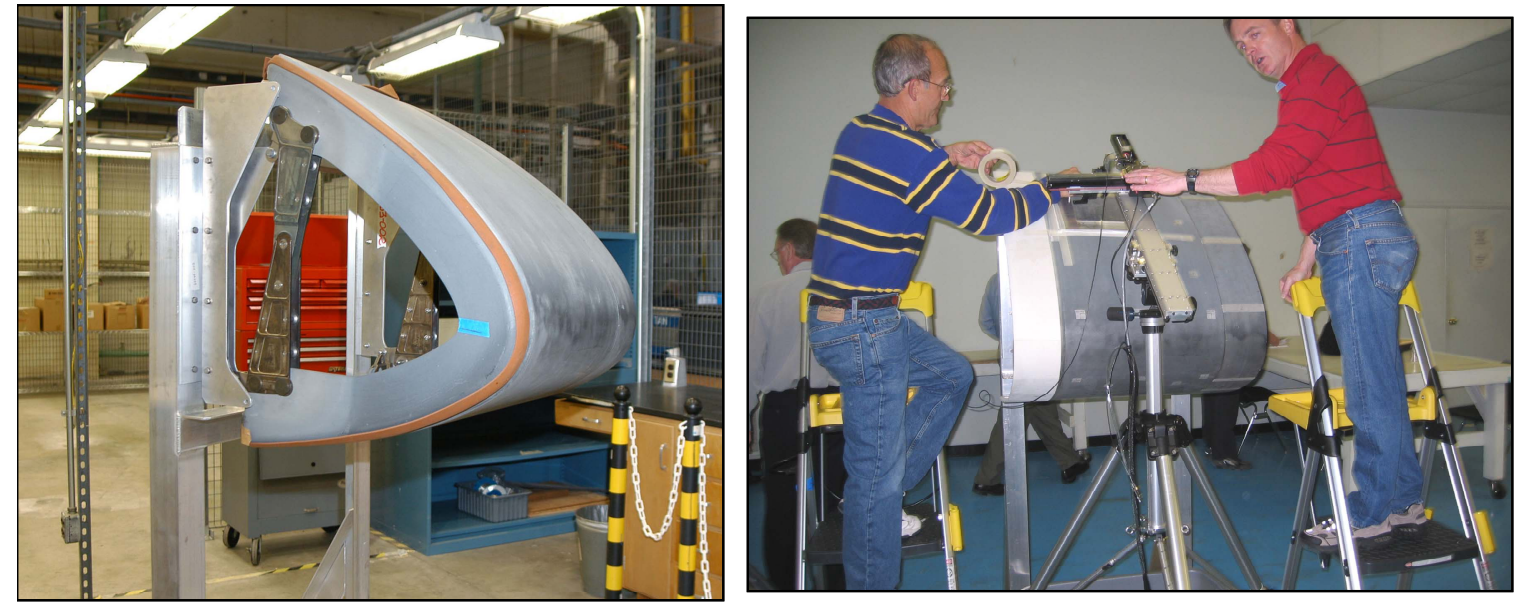

Figure 14: Final Validation and NASA Certification Tests Using RCC Heat Shields Mounted in a Mock-Up of the Wing Leading Edge Configuration

Figure 14 also shows the blind inspections in process with the full deployment of all UT hardware described above. It was determined that the scanner device, coupled with the linear slide and tripod mount, was able to access all inspection regions on the test panels. After each C-scan was acquired, the data was analyzed using the time-of-flight and amplitude data reduction methods described above. The knowledge gained from all of the inspections completed prior to the blind tests, was used to set the series of UT gates for optimum data. The location and size of all detected flaws were provided to the NASA test monitors along with annotated C-scan images which showed the presence of the flaws.

Final UT Inspection Results - Recall that the inspection requirement was to reliably detect $1 / 4$ " diameter flaws while producing minimal false calls that would require additional investigation. A statistical analysis was performed on all inspection results to produce Probability of Detection (POD) curves [5-6]. Of the 77 flaws that were included in the blind certification tests, 66 were detected (86\%). None of the missed flaws were larger than $1 / 8$ ” in 
diameter. The resulting flaw POD curve revealed that the required 90\% POD level occurred at a flaw size of 0.22 " in diameter. Figure 15 is the POD curve that was produced from all of the validation testing that was completed during the study. The probability of flaw detection is plotted versus the size of the flaw in the RCC panels. A total of 46 different test specimens were inspected with the UT system. They contained 306 flaws of representative origin, size, shape, and location. The pulse-echo UT inspection system detected 257 of the 306 flaws. Forty-five of the 49 undetected flaws (92\%) were 1/8" in diameter (i.e. below the required detection level). Thus, $98 \%$ of flaws larger than 1/8" dia. were detected. The POD curve in Figure 15 shows that the overall 90\% POD level for the UT inspection method occurs at a flaw size of less than $1 / 4$ " in diameter (Comprehensive $90 \%$ POD $=0.21$ ” dia.). Another important performance metric is that these POD levels were attained without producing any false calls.

\section{UT Validation PoD - All NASA RCC Specimens}

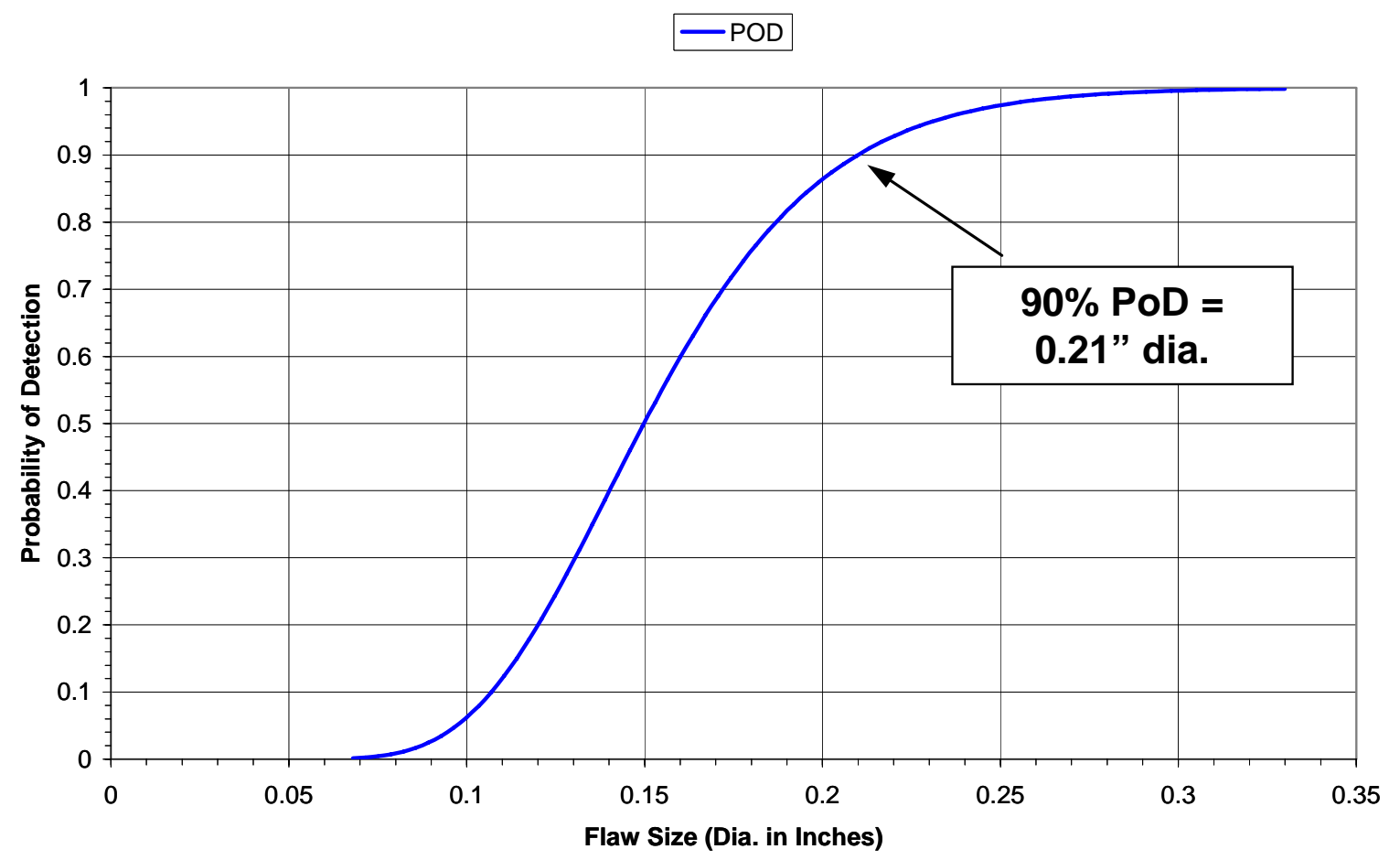

Figure 15: Probability of Detection Curve Representing the Flaw Detection Capability for the Pulse-Echo Ultrasonic Inspection Method as Calculated from the Results of All NASA NDI Validation Specimens

\section{Conclusions}

The reinforced carbon-carbon (RCC) heat shield components on the Space Shuttle's wings must withstand harsh atmospheric reentry environments where the wing leading edge can reach temperatures of $3,000^{\circ} \mathrm{F}$. Potential damage includes impact damage, micro cracks, oxidation in the silicon carbide-to-carbon-carbon layers, and interlaminar disbonds. Since accumulated damage in these structures can lead to catastrophic failure of the Shuttle's heat protection system, it was imperative for NASA to institute a nondestructive inspection program that could certify the integrity of the Shuttle wing heat shields. The pulse-echo ultrasonic inspection method and hardware system described in this paper possesses the accuracy, sensitivity, reliability, and deployment capabilities needed to properly monitor the 
health of the RCC panels. Flight hardware and scrap heat shield panels were used to discover and overcome the obstacles associated with damage detection in the RCC material. Optimum combinations of custom ultrasonic probes and data anlaysis were merged with the inspection procedures needed to properly survey the heat shield panels. System features were introduced to minimize the potential for human factors errors in identifying and locating the flaws. Extensive validation testing proved that the pitch-catch inspection system was capable of detecting the required 1/4" diameter flaws anywhere within the thickness of the RCC panels. In addition, the UT device met the other NDI requirements including weight restrictions, ease of deployment to inspect the heat shields while they are still mounted on the Orbiter, and ability to access all regions and navigate all contours on the RCC panels. The ultrasonic inspection system is now in use at NASA to complete "Return-to-Flight" certification inspections prior to each Shuttle launch.

\section{References}

[1] NASA Columbia Accident Investigation Board, "NASA’s Implementation Plan for Return to Flight and Beyond,” NASA Report, Sept. 2003.

[2] Roach, D, Rackow, K., "Pulse-Echo Ultrasonic Inspection System for In-Situ Nondestructive Inspection for Space Shuttle RCC Heat Shields,” Dept. of Energy SAND Report 2005-3429, June 2005.

[3] Beattie, A., Dahlke, L., Gieske, J., Hansche, B., Phipps, G., Roach, D., Shagam, R., and Thompson, K., "Emerging Nondestructive Inspection for Aging Aircraft," Dept. of Transportation Report No. DOT/FAA/CT-94/11, October 1994.

[4] Gieske, J.H., "Evaluation of Scanners for C-Scan Imaging in Nondestructive Inspection of Aircraft," Dept. of Energy Sandia Report, SAND94-0945, April 1994.

[5] Berens, A., "NDE Reliability Data Analysis," ASM Handbook, 9 ${ }^{\text {th }}$ Ed., vol. 17, Metals Park, Ohio, ASM International, 1989.

[6] Spencer, F., "Fitting Probability of Detection Curves to Single Inspector Hit/Miss Data," Air Transport Assoc. NDT Forum, 1998. 